NEPHRON

Nephron 1998;78:116

\section{Olivier Devuyst}

Division of Nephrology, St. Luc Academic Hospital, University of Louvain Medical School, Brussels, Belgium

\title{
The Expression of Water Channels AQP1 and AQP2 in a Large Series of ADPKD Kidneys
}

analysis in 4 ADPKD kidneys (991 randomly selected cysts were examined) to evaluate AQP1 and AQP2 immunolabeling as a function of the size of cysts. This analysis showed that total water channel expression decreased with increasing cyst size, in relation with a significant, size-related decrease in AQP1 expression, whereas the percentage of AQP2-positive cysts did not alter significantly. Consequently, the ratio of AQP2- to AQP1-positive cysts increased significantly with cyst enlargement. The expression of AQP1 and AQP2 in ADPKD was confirmed by Western blot analysis, which also showed a decrease in AQP expression associated with the degree of cystic progression in ADPKD.

In conclusion, our data confirm and extend those presented by Hayashi et al. [1]. From the quantification of AQP1 and AQP2 immunoreactivity in a total of 991 ADPKD cysts, we confirm that a mean of $33 \%$ of cysts stained for AQP2, while a mean of $30 \%$ of cysts stained for AQP1. Expression of AQP1 and AQP2 are mutually exclusive, even in end-stage ADPKD, when fine morphological discrimination of tubular origin is lost. The fact that one third of the cysts express neither AQP1 nor AQP2 might imply that these cysts originate from nephron segments that do not express these channels, e.g. the ascending limb of Henle's loop, and the connecting and distal convoluted tubules. Thus, aquaporins 1-3 appear to belong to a group of proteins (such as GP330 or vinculin) [3] that retain their differential expression even into end-stage ADPKD.

\section{References}

1 Hayashi M, Yamaji Y, Monkawa T, Yoshida $\mathrm{T}$, Tsuganezawa H, Sasamura H, Kitajima W, Sasaki S, Ishibashi K, Marumo F, Saruta T: Expression and localization of water channels in human autosomal dominant polycystic kidney disease. Nephron 1997;75:321-326.

2 Devuyst O, Burrow CR, Smith BL, Agre P, Knepper MA, Wilson PD: Expression of aquaporins- 1 and -2 during nephrogenesis and in autosomal dominant polycystic kidney disease. Am J Physiol 1996;271:F169-F183.

3 Wilson PD, Falkenstein D: The pathology of human renal cystic disease; in Dodd SM (ed): Current Topics in Pathology. Heidelberg, Springer, 1995, pp 1-50.

\section{KARGER \\ Fax +4161306 1234 E-Mail karger@karger.ch} www. karger.com
(C) 1998 S. Karger AG, Basel

0028-2766/98/0781-0116\$15.00/0

This article is also accessible online at: http://BioMedNet.com/karger
Olivier Devuyst, MD

Division of Nephrology, St. Luc Academic Hospital

University of Louvain Medical School

10 Avenue Hippocrate, B-1200 Brussels (Belgium)

Tel. +32 2764 1857, Fax +32 2764 2836, E-Mail devuyst@nefr.ucl.ac.be 